Meta

Journal des traducteurs

Translators' Journal

\title{
The four-letter word, ou comment traduire les mots fuck et fucking dans un texte littéraire?
}

\section{Julie Adam}

Volume 43, numéro 2, juin 1998

URI : https://id.erudit.org/iderudit/001852ar

DOI : https://doi.org/10.7202/001852ar

Aller au sommaire du numéro

Éditeur(s)

Les Presses de l'Université de Montréal

ISSN

0026-0452 (imprimé)

1492-1421 (numérique)

Découvrir la revue

Citer cet article

Adam, J. (1998). The four-letter word, ou comment traduire les mots fuck et fucking dans un texte littéraire ? Meta, 43(2), 236-241.

https://doi.org/10.7202/001852ar

\section{Résumé de l'article}

Principale difficulté de traduction de la nouvelle A Summer Girl, de John McKenna, les mots fuck et fucking apparaissent 34 fois dans un texte d'environ 8900 mots. Nous avons puisé dans le vocabulaire oral pour rendre la charge affective et esthétique de ces deux mots, emblématiques des sentiments de haine et d'amour qu'éprouve la narratrice envers son interlocuteur. Pour reproduire un effet similaire à celui du texte original, il fallait étudier les diverses valeurs des mots fuck et fucking selon le contexte. On distingue quatre grands types d'emploi : sens littéral, injure, formules marquant la colère, le mépris ou l'amertume et enfin formules d'insistance. 


\title{
THE FOUR-LETTER WORD, OU COMMENT TRADUIRE LES MOTS FUCK ET FUCKING DANS UN TEXTE LITTÉRAIRE?
}

JULIE ADAM

Université Laval, Québec, Canada

\begin{abstract}
Résumé
Principale difficulté de traduction de la nouvelle A Summer Girl, de John McKenna, les mots fuck et fucking apparaissent 34 fois dans un texte d'environ 8900 mots. Nous avons puisé dans le vocabulaire oral pour rendre la charge affective et esthétique de ces deux mots, emblématiques des sentiments de haine et d'amour qu'éprouve la narratrice envers son interlocuteur. Pour reproduire un effet similaire à celui du texte original, il fallait étudier les diverses valeurs des mots fuck et fucking selon le contexte. On distingue quatre grands types d'emploi : sens littéral, injure, formules marquant la colère, le mépris ou l'amertume et enfin formules d'insistance.
\end{abstract}

\begin{abstract}
John McKenna's short story A Summer Girl raises the problem of how to translate the words fuck and fucking, occurring 34 times in a text of about 8,900 words. In order to convey the emotional and aesthetic power of these two words, emblematic of the narrator's feelings of love and hatred, we looked at oral expressions in Quebec French. Since the main goal is to reproduce the same effect as the original text, we examined the various meanings of the words fuck and fucking according to their contexts. Four main nuances emerge from the analysis: literal meaning, insult, anger, contempt or bitterness, and emphasis.
\end{abstract}

Les particularités du vocabulaire québécois par rapport au français de l'Hexagone ou au français dit «international» placent parfois le traducteur devant un choix inconfortable : quelle variété de français doit-il privilégier lorsqu'il s'adresse à un public francophone relativement indéterminé ? Souvent, seuls quelques mots comme ice cream, lunch, dinner ou weekend marquent la différence. Le choix peut paraître alors plus aisé, dans la mesure où ces mots ne sont pas légion dans un texte et que certains d'entre eux admettent des équivalents neutres comme «manger un morceau», «casser la croûte», «le repas», etc. Cependant, les niveaux de langue populaire et vulgaire imposent un choix sans équivoque. C'est ce que nous avons constaté en traduisant un recueil de nouvelles irlandaises contemporaines. L'une de ces nouvelles, A Summer Girl', de l'auteur John McKenna, met en lumière le défi que représente la traduction d'expressions vulgaires telles que bastard, shit, bloody, half-arsed, pissed, pissed out, dick, frigging, screw, bollocks, fuck et fucking. Il s'agit d'un texte marqué par l'oralité, où dominent la colère et des cris de haine mêlée d'amour : une femme se vide le cœur et raconte son aventure avec un homme qui vient de mourir noyé. Au fil du texte, nous comprenons que le personnage principal vivait de prostitution avant de rencontrer cet homme, un genre de prédicateur qui lui aura fait miroiter l'espoir d'un amour. La nouvelle est en fait un long discours ininterrompu, si ce n'est les quatre citations bibliques placées en exergue de chaque chapitre, discours par lequel la narratrice s'adresse 
au mort comme si elle lui parlait de vive voix : emploi du you, nombreuses ellipses du verbe ou du sujet, interrogations directes, formes contractées de verbes, d'adverbes et d'auxiliaires (par exemple I'd, mightn't, that's, didn't), digressions fréquentes, etc.

L'une des principales difficultés de traduction tient à l'emploi répété des mots fuck et fucking, qui apparaissent 34 fois dans un texte d'environ 8900 mots. La difficulté est d'autant plus importante que ces deux petits mots symbolisent à eux seuls la charge affective et esthétique qui traverse le texte. C'est en effet par des expressions comme fuck you que s'exprime le déchirement de la narratrice, partagée entre l'amour et la haine qu'elle éprouve envers le prédicateur.

Outre le verbe «baiser» et son dérivé - peu usité, nous semble-t-il — «baisage», que propose le dictionnaire Robert \& Collins Senior sous l'entrée fuck? Par exemple, fuck it devient «putain de merde» et fuck you, «va te faire foutre». Quant aux expressions formées à partir de fucking, elles sont rendues par des mots ou locutions comme «vachement», «putain de merde», «foutu», «foutrement», «nom de Dieu», «bordel», etc. Bien que le cinéma américain doublé en France nous ait habitués à ce genre d'expressions, force est de constater qu'elles n'ont jamais réellement pénétré le parler québécois. S'il est vrai que certaines locutions comme «espèce de salaud», «mon cul» ou «complètement con» franchissent plus aisément les continents de la francophonie, il reste que la colère populaire ne s'exprime généralement pas de la même façon en Europe et en Amérique. Ainsi, les références sexuelles sont réduites à la portion congrue au Québec, supplantées par les répertoires scatologique et, surtout, religieux. L'importance du religieux est telle, d'ailleurs, que nombre de dérivés ont été formés à partir des blasphèmes : «ostiment», «crisser» et «décrisser» témoignent de cette créativité...

Le dictionnaire bilingue offrant peu de ressources utiles pour ce qui est des jurons québécois, nous avons puisé dans le vocabulaire oral pour rendre le poids de l'outrage exprimé en anglais. Comme il est impossible de proposer un équivalent unique pour ces nombreux fuck et fucking, nous avons traité chacun des cas selon le contexte. La reprise systématique d'un même mot aurait en effet semblé lourde en français, tandis que l'anglais tolère très bien ces répétitions. L'essentiel étant de reproduire un effet similaire à celui qu'exerce le texte original sur le lecteur anglophone, il fallait donc étudier les diverses valeurs que prennent les mots fuck et fucking dans la phrase et, plus largement, dans l'économie du texte. L'étude des différentes occurrences de ces deux mots permet de dégager quatre grands types d'emploi. (Voir tableau.)

Le sens premier de fuck est bien sûr présent dans la nouvelle, par exemple lorsque la narratrice mentionne ses activités professionnelles ou qu'elle exprime son amertume à l'égard de celui qui l'a traitée non pas comme une amoureuse mais comme un objet sexuel. On trouve sept exemples de ce type d'emploi dans A Summer Girl, dont les passages suivants :

I'd make love to you. I never fucked you. [...] But you were fucking me, the same as the others did. You just put a polished name on it.

Je te faisais l'amour, moi. J'ai jamais baisé avec toi. [...] Mais toi, tu baisais avec moi. Tu étais pas différent des autres, sauf que tu avais un beau mot pour ça.

She liked him. I don't mean to fuck. Just liked him.

Elle l'aimait bien. Je veux pas dire pour baiser. Juste comme ça.

I'd say nothing while I was being fucked, unless I was paid to talk.

Je disais rien pendant qu'on baisait avec moi, à moins qu'on m'ait payée pour parler.

Dans tous ces cas, nous avons donc utilisé l'équivalent «baiser», sauf dans une phrase où la forme nominale «baise» (jugée plus appropriée que «baisage») traduit 
fucking : Not fucking around yet but on my way to it devient ainsi «Pas de baise encore mais ça s'en venait».

Par ailleurs, le verbe fuck peut également prendre valeur d'injure, notamment lorsqu'il est suivi du pronom you. Cinq phrases illustrent cet aspect dans notre nouvelle. Plutôt que d'opter pour une formule du type «va te faire foutre», nous avons cru bon de choisir une insulte qui, tout en heurtant l'oreille par sa vulgarité, apparaisse plus spontanée, et donc plus vraisemblable pour un lecteur québécois. C'est pourquoi trois de ces passages sont traduits par l'expression «va chier»; quant aux deux autres, ils sont rendus respectivement par l'apostrophe «mon salaud!» et la phrase nominale «Une vraie tête enflée». Bien que ce dernier choix puisse apparaître fort éloigné de la phrase anglaise, il demeure fidèle à l'esprit de la nouvelle : la narratrice ne cesse en effet d'illustrer l'égoïsme et la suffisance de celui qui prétend guérir l'humanité alors qu'il reste insensible, dans les faits, à la misère des individus venus écouter ce que la narratrice juge comme «des paroles creuses» — paroles qui ne font que flatter l'oreille du prédicateur. On pourra objecter qu'il s'agit d'une interprétation de notre part, mais elle nous semble justifiée dans la mesure où elle respecte le ton de l'auteur et contribue à préserver la couleur du texte sans verser dans la répétition. De même, si l'apostrophe «mon salaud !» ajoute un élément d'information qui ne se trouve pas dans l'expression fuck you, il s'agit toutefois d'une injure qui s'insère très bien dans le passage en question, étant donné que la narratrice ne manque pas de traiter son «interlocuteur» de bastard à quelques reprises. Il s'agit par conséquent d'un ajout qui tient compte de l'ensemble du texte, plus précisément du discours et des caractéristiques des personnages. Voici donc un exemple de chaque traduction :

I was looking better than I ever did. I know I was. I felt it. Fuck you.

J'avais jamais été aussi élégante. Je sais que je l'étais. Je le sentais. Va chier.

This time you're going to listen. Wherever you are. You're going to listen. Listen to me, fuck you.

Tu vas m'écouter, cette fois-ci. Où que tu sois. Tu vas m'écouter. Écoute-moi, mon salaud !

You remember the blue frock I had. The one you said made me look like the sun on the sea. Fuck, you said that.

Tu te souviens de mon manteau bleu. Celui qui t'a fait dire que je ressemblais au soleil sur la mer. Va chier, c'est exactement ce que tu as dit.

I could look at men that came to you and I could see their lives immediately. [...] I could see them driving like fuck to get to you in Naas or Newbridge or Baltinglass, to hear you, to believe in you, to be cured of something there's no cure for. I could see that, as soon as they came into the hotels. You saw nothing. Fuck you.

Je regardais les hommes qui venaient te rencontrer et je pouvais tout de suite voir leur vie. [...] Je les voyais conduire en fou pour aller te voir à Naas ou Newbridge ou Baltinglass, pour t'entendre, pour te croire, pour se faire guérir d'une souffrance inguérissable. Je voyais tout ça dès qu'ils entraient dans la salle. Toi, tu voyais rien. Une vraie tête enflée.

Les deux autres catégories regroupent la majeure partie des occurrences de fuck et fucking, soit 22 emplois de l'un ou l'autre des termes. Le troisième groupe comprend toutes les formules à valeur adjectivale marquant la colère, le mépris ou encore l'amertume. En somme, il s'agit de passages où l'injure est soit absente, soit moins importante que ces sentiments. Par exemple, la narratrice parle d'un petit garçon d'origine modeste qu'elle admire pour la vie ordinaire, presque anonyme, qu'il a menée et la façon, tout aussi discrète, dont il est mort au terme d'une agonie causée par les flammes :

That's what I'd call a hero, that's what I'd say. But then he never said anything clever, did he? He didn't get a fucking chance to, did he? 
C'est ça que moi j'appelle un héros. Mais il a jamais rien dit de brillant, hein ? Il a jamais eu une crisse de chance de faire son fin finaud.

L'utilisation du juron «crisse» sert ici à souligner l'amertume et la colère que ressent la narratrice, révoltée à l'idée que l'on puisse considérer le prédicateur comme un héros tandis que personne ne s'émeut de la mort du jeune garçon : pourquoi réserver les honneurs aux hommes apparemment brillants, alors que nombre de gens ordinaires affrontent la misère quotidienne avec grand courage ? Le choix du mot «crisse» parmi tant d'autres possibilités nous semblait d'autant plus indiqué qu'il s'agit d'un blasphème particulièrement offensant. Ainsi, le personnage crache son mépris non seulement pour l'amoureux déloyal mais aussi pour la mission spirituelle à laquelle il prétendait :

Everywhere you went you dragged that frigging pulpit behind you, that fucking pulpit, you weren't happy when you weren't up there, were you?

Partout où tu allais, il fallait que tu traînes ta maudite chaire avec toi, ta crisse de chaire, tu étais pas content ailleurs qu'en chaire, pas vrai ?

You had them to adore you, to worship at your fucking shrine, hadn't you?

Tu t'es arrangé pour qu'ils te lèchent les bottes, pour qu'ils adorent ton crisse de tombeau, pas vrai?

You could only see your own hands. You couldn't see beyond the hands to the heads that were under them, the limp bodies, the smashed-up fucking hearts that would have done anything to be part of something, part of your circle.

Tu voyais seulement tes mains. Tes mains mais pas la tête qui était en-dessous, pas le corps sans force, pas le cœur décrissé qui aurait fait n'importe quoi pour appartenir à quelque chose, à ton petit milieu.

On constate que, dans ce dernier exemple, l'adjectif «décrissé» traduit à la fois smashed-up et fucking. Il faut cependant noter que nous avons eu recours à d'autres solutions que le seul mot «crisse» ou l'un de ses dérivés pour rendre la valeur d'emploi associée à l'amertume, au mépris ou à la colère. Par exemple, l'adjectif «maudit» et l'expression populaire «s'en ficher comme de l'an quarante» traduisent dans l'extrait suivant l'amertume de la femme à la suite d'une aventure qui ne lui aura valu, de la part du prédicateur, que mépris et indifférence.

Love is a fucking waste of time. It won't get you loved if there isn't love on the other side. I could do anything and it'd mean fuck all to you.

L'amour, c'est rien qu'une maudite perte de temps. Ça sert à rien quand c'est pas réciproque. J'aurais pu faire n'importe quoi, mais tu te fichais de moi comme de l'an quarante.

Parmi les divers choix possibles, celui de ne pas traduire nous est apparu légitime dans le cas d'un passage particulièrement «lourd» : n'en jetons plus, la cour est pleine...

At least that guy that paid me to come in my hair needed that. He needed me for that. He was willing to put his money where his fucking dick was.

$\mathrm{Au}$ moins, le type qui me payait pour venir dans mes cheveux avait besoin de ça. Il avait besoin de moi pour ça. Il était prêt à mettre son argent à la même place que sa bitte.

Or, l'omission n'est que rarement possible lorsqu'il s'agit de reproduire un effet équivalent à celui du texte original. L'autre solution privilégiée pour traduire le mépris ou la colère met en jeu le procédé de transposition tel que l'ont décrit Vinay et Darbelnet. À deux endroits, l'adjectif fucking est rendu par un mot de nature grammaticale différente, soit le verbe «déblatérer» et la locution «crisser son camp»:

The whisperer never saw you like that. If he did he wouldn't go on with that fucking rubbish about your body being cleansed in the river that weekend. 
Le souffleur t'a jamais vu comme ça. S'il t'avait vu, il arrêterait de déblatérer ses niaiseries sur la purification de ton corps par l'eau.

I just got my fucking coat or put on my clothes and left.

Je prenais tout simplement mon manteau ou je me rhabillais, pis je crissais mon camp.

Enfin, les mots fuck et fucking remplissent parfois une fonction adverbiale ou adjectivale d'insistance. Dans certaines phrases, en effet, le poids sémantique de ces mots semble occulté par leur valeur affective et rhétorique : ils deviennent alors figures de rythme en ce qu'ils appuient une répétition et facilitent la cohésion du discours.

Don't ask me for logic, for a straight story, don't fucking ask me for anything like that.

Demande-moi pas d'être logique, de te raconter une histoire cohérente, demande-moi surtout rien de ça.

He knew what he should do. He fucking knew what was right.

Il savait ce qu'il avait à faire. Il savait pas mal plus que toi ce qui était correct.

Comme on le voit dans les exemples précédents, le verbe auquel se rapporte fucking est essentiellement souligné par ces derniers, sans que soit nécessairement exprimée quelque idée d'agressivité. Certes, les mots fuck et fucking participent dans l'ensemble au ton accusateur de la nouvelle et demeurent, par leur vulgarité indéniable, agressants; toutefois, ils deviennent en quelque sorte des termes passe-partout dans certains passages : vidés de leur contenu sémantique, ils occupent la place des divers adjectifs ou adverbes qu'un autre narrateur aurait peut-être jugé approprié d'employer pour insister sur tel propos ou tel sentiment. C'est d'ailleurs dans cette dernière catégorie que l'on trouve le plus de diversité quant aux choix de traduction, puisqu'il s'agissait dans ces cas de renforcer chaque phrase ou expression selon son contexte propre. La valeur d'insistance s'exprime ainsi de plusieurs façons, tantôt par des phrases courtes, elliptiques, tantôt par des figures telles que l'ironie ou l'oxymore, comme en témoignent respectivement les trois exemples suivants :

Course I'm in a state of shock but not the one you think. No fucking chance. None.

C'est sûr que je suis en état de choc, mais pas celui que tu penses. Pas pantoute. Aucun rapport.

You said there wasn't enough minutes in the day to be with me. Too fucking true, sunshine. Tu as dit qu'il y avait pas assez de minutes dans une journée pour rester avec moi. Ben sûr, mon rayon de soleil.

No one could ever say I didn't know how to leave in my time. When it was up I knew and I'd go with some... some... some fucking grace.

Personne pourra jamais dire que je savais pas m'en aller au bon moment. Quand c'était fini, je le savais et je m'en allais avec... avec... une maudite belle élégance.

Les traductions des mots fuck et fucking appartenant à la quatrième catégorie font donc appel avant tout aux connotations affectives du vocabulaire français. La principale difficulté consistait ainsi à trouver des équivalents qui, tout en rendant l'intensité affective du mot anglais, apparaissent aussi spontanés, aussi idiomatiques dans le texte français, texte «prononcé» par une femme qui, semble-t-il, ne possède pas un vocabulaire très étendu.

Notre tentative de classification des divers types d'emploi de fuck et fucking ne saurait masquer le fait que les frontières entre l'injure, la valeur d'emploi marquant la colère, l'amertume ou le mépris et enfin la valeur d'insistance demeurent discutables. D'ailleurs, il s'agit là d'une classification réalisée a posteriori : ce n'est qu'après avoir traduit, de façon plutôt intuitive, cette nouvelle de John McKenna qu'est venue l'idée d'une schématisation des réponses apportées à une difficulté persistante. Comme nous l'avons constaté, les mots 
fuck et fucking sont polysémiques et témoignent du déchirement de la narratrice, des sentiments troubles qu'elle éprouve à l'égard d'un homme à la fois adoré et détesté. Cette ambiguïté même explique en partie la fragilité de toute classification. Mais les frontières sont également brouillées par le fait des relations étroites entre injure d'une part et, d'autre part, colère et mépris - l'injure s'accompagne naturellement de colère ou de mépris, la colère ou le mépris suscitent l'injure... — ou encore entre le sentiment d'amertume et la façon dont celle-ci peut être soulignée. Par exemple, dans la phrase Love is a fucking waste of time, le mot fucking exprime l'amertume tout en servant une fonction d'insistance. Malgré les zones grises, cette classification nous aura néanmoins permis de réfléchir sur la pertinence des divers choix de traduction privilégiés dans notre nouvelle. Elle confirme également l'idée selon laquelle un même effet esthétique ne s'exprime pas nécessairement, tant s'en faut, de la même façon d'une langue à l'autre. En l'occurrence, il nous semblait impératif d'emprunter de multiples avenues pour rendre justice à deux seuls mots; d'où l'importance de considérer l'ensemble du texte - ton, propos, caractéristiques des personnages, couleur du vocabulaire - pour traduire chacun des passages particulièrement critiques.

\section{Note}

1. Ce texte, traduit sous le titre «Un amour d'été», ainsi que les autres nouvelles formant le recueil Nouvelles d'Irlande paraîtront en 1996 aux Éditions L'instant même. Traduction de Louis Jolicoeur et Julie Adam. 